\title{
Human ether-à-go-go-related gene mutation L539fs/47-hERG leads to cell apoptosis through the endoplasmic reticulum stress pathway
}

\author{
SHUTING MA, YUN ZHAO, MIAOMIAO CAO and CHAOFENG SUN \\ Department of Cardiovascular Medicine, First Affiliated Hospital of Xi'an Jiaotong University, \\ Institute of Cardiovascular Channelopathy, Key Laboratory of Molecular Cardiology, Xi'an, Shaanxi 710061, P.R. China
}

Received June 3, 2018; Accepted December 31, 2018

DOI: $10.3892 / \mathrm{ijmm} .2019 .4049$

\begin{abstract}
Congenital long QT syndrome (LQTS) is a cardiac channelopathy that often results in fatal arrhythmias. LQTS mutations not only lead to abnormal myocardial electrical activities but are associated with heart contraction abnormalities, cardiomyopathy and congenital heart defects. In vivo and in vitro studies have found that LQTS mutations are associated with cardiomyocyte apoptosis, cardiac developmental disorders and even embryonic mortality. Cardiac delayed rectifier potassium channel dysfunction due to the human ether-à-go-go-related gene (hERG) mutation causes congenital LQTS type 2. The majority of LQTS 2 mutations are characterized by mutant protein accumulation in the endoplasmic reticulum (ER). Unfolded or misfolded protein retention in the ER causes an unfolded protein reaction, which is characteristic of ER stress (ERS). Therefore, the present study hypothesized that LQTS mutations can cause cardiac structural abnormalities via ERS-mediated cardiomyocyte apoptosis. To test this hypothesis, 293 cells were transiently transfected with an L539fs/47-hERG plasmid to generate an LQTS 2 model. L539fs/47-hERG is an LQTS 2 mutation, which consists of a 19-bp deletion at 1619-1637 and a point mutation at 1692 . Using confocal laser scanning microscopy analysis, it was verified that the L539fs/47-hERG protein was retained in the ER. Hoechst 33342 apoptosis staining indicated that apoptosis was increased in the L539fs/47-hERG-transfected cells, and this be reversed by treatment with 4-phenyl butyric acid. Western blot analysis
\end{abstract}

Correspondence to: Professor Chaofeng Sun, Department of Cardiovascular Medicine, First Affiliated Hospital of Xi'an Jiaotong University, Institute of Cardiovascular Channelopathy, Key Laboratory of Molecular Cardiology, 277 Yanta West Road, Xi'an, Shaanxi 710061, P.R. China

E-mail: cfsun1@mail.xjtu.edu.cn

Key words: congenital long QT syndrome, human ether-à-go-go-related gene, gene mutation, structural abnormality, apoptosis, endoplasmic reticulum stress revealed increased expression levels of the ERS chaperone glucose regulated protein 78 and pro-apoptotic ERS-induced factors, including protein kinase R-like endoplasmic reticulum kinase, eukaryotic translation-initiation factor- $2 \alpha$ and C/EBP homologous protein, in the L539fs/47-hERG-transfected cells. The B-cell lymphoma (Bcl-2)-associated X protein/Bcl-2 ratio and caspase-12 were also increased in the mutated cells. These results demonstrate that L539fs/47-hERG induces cell apoptosis and the potential molecular mechanism involves the activation of ERS and ERS-mediated cell apoptosis.

\section{Introduction}

Congenital long QT syndrome (LQTS) is a series of conditions caused by cardiomyocyte ion channel mutations. Among the 15 types of LQTS, types 1, 2 and 3 are the most common and account for $95 \%$ of all congenital LQTS cases $(1,2)$. Cardiac electrical activity disorders are the most notable characteristics of LQTS, however, structural abnormalities of the heart in patients with LQTS, which have been identified in several previous studies, have long been ignored. In the early 1990s, Nador et al found that $~ 55 \%$ of patients with LQTS had ventricle contraction abnormalities (3). Of 216 patients with LQTS at the Mayo Clinic (Rochester, MN, USA), 25\% had echocardiography abnormalities (4). The high detectability of LQTS-associated gene mutations in certain cardiomyopathies, including arrhythmogenic right ventricular cardiomyopathy, dilated cardiomyopathy, left ventricular noncompaction and ventricular remodeling, show that the gene mutations of LQTS-associated channels are important in these cardiomyopathies (5). Several types of infant congenital heart defects are also accompanied by the detection of LQTS-associated gene mutations $(6,7)$. These results demonstrate that LQTS structural abnormalities are not individual cases but show prevalence in the LQTS patient population. However, these abnormalities have not been recognized by clinicians.

As reported in the aforementioned studies, the structural changes of the heart in patients with LQTS cannot be explained completely by abnormal electrical activity, such as a prolonged repolarization time or ventricular tachyarrhythmia. The mechanisms underlying structural changes of LQTS have been examined previously. The most common accepted hypothesis 
is that LQTS gene mutations can induce cell apoptosis. In 1993, James et al observed abnormal cell apoptosis in biopsies of vascular endothelium cells, vascular smooth muscle cells, sinoatrial node cells and cardiomyocytes around the node obtained from patients with LQTS (8). In a previous in vivo study, N1325S-SCN5A transgenic mice were found to manifest cardiac fibrosis and partial contraction disability, which was mediated by ventricle cardiomyocyte apoptosis (9). Teng et al demonstrated that N629D-hERG homozygous transgenic mice exhibited cardiomyocyte apoptosis and cardiac deformity, and fetal mortality within 11 days (10). These findings suggest that the mechanism underlying the structural abnormalities of LQTS may involve cell apoptosis caused by LQTS-related gene mutations.

Endoplasmic reticulum stress (ERS) has a significant role in defending against or adapting to cellular damage in order to restore homeostasis. The unfolded protein response (UPR) is the most widely investigated pathway in ERS. The UPR can be triggered by large quantities of unfolded or misfolded proteins that have accumulated in the ER; this results in ERS-associated proteins, such as glucose regulated protein 78 (GRP78), being upregulated, decreased whole-cell scale protein expression, or ER-associated degradation (11). The UPR is composed of three downstream signal transduction pathways: Protein kinase R-like endoplasmic reticulum kinase (PERK), activating transcription factor 6 (ATF6) and inositol-requiring enzyme 1 (IRE1). When ERS occurs, the expression of GRP78 increases, and it dissociates from PERK, ATF6 or IRE1 so it can recognize and assist in the folding of any misfolded proteins or in degrading the misfolded protein. If the ERS is persistent or excessive, and the cell cannot be rescued from damage, then programed cell death, particularly cell apoptosis, is initiated. PERK-eukaryotic translation-initiation factor- $\alpha \alpha$ (eIF2 $\alpha$ )-C/EBP homologous protein (CHOP) is a significant ERS-mediated apoptotic pathway. PERK can be phosphorylated to activate eIF2 $\alpha$ and promote the expression of CHOP/GADD153, which is an important apoptosis-inducing transcription factor (12). B-cell lymphoma 2 (Bcl-2) and Bcl-2-associated X protein (Bax) are a pair of molecules that have anti-and pro-apoptotic regulatory effects, respectively (13). They are also involved in the regulation of ERS-mediated cell death (14). One of the caspase members, caspase-12, is an ER-specific protein that can be activated under ERS conditions. Cleaved caspase-12 can activate and initiate downstream enzyme reactions, ultimately starting the process of apoptosis (15). The activation of caspase-3 is the terminal step of cell apoptosis; it can be cleaved to its activated form to complete apoptosis (16).

The human ether-à-go-go-related gene (hERG) encodes the hERG channel, which produces the important repolarization current $\mathrm{I}_{\mathrm{Kr}}$. Mutations of this channel lead to channel dysfunction and result in LQTS type 2. The majority of the hERG mutations are characterized by channel protein transfer deficiency, with protein accumulating in endoplasmic reticulum (ER) and a failure of the channel to anchor in the cell membrane as a functional ion channel (17). It has been found that mutated I539R-hERG protein accumulates in the ER, activating ERS through the ATF6 pathway (18). The same effect occurs with unfolded E637R-hERG and G572R-hERG proteins, which are degraded by activating ERS-induced proteasome degradation (19). Mutations of hERG can cause protein retention in the ER and evoke the UPR; whether this is the mechanism of LQTS 2-induced cardiomyocyte apoptosis requires further investigation.

Based on the evidence described above, it was hypothesized that LQTS $2 \mathrm{hERG}$ mutations cause cell apoptosis by inducing the ERS pathway. In the present study, the LQTS 2 family mutation L539fs/47-hERG was used. It was found that L539fs/47-hERG is a complex mutation consisting of a 19-bp deletion at site 1619-1637 (CCGTACTCTGAGTAGCGAT) together with an $A \rightarrow G$ point mutation at $1692 \mathrm{bp}$, resulting in a frame-shift after the 539th amino acid and a premature stop at the 47 th amino acid after this. The mutated hERG protein translation ends at the 4 th transmembrane section. It was also found that this mutation results in truncated protein transfer deficiency, protein retention in the ER and dysfunction of the hERG channel (20). The L539fs/47-hERG plasmid was used to construct an LQTS 2 cell model in the present study. This was used to investigate cell apoptosis and detect the expression levels of ERS-associated factors to identify possible molecular pathways involved in ERS-induced apoptosis.

\section{Materials and methods}

Plasmid propagation and DNA sequencing. The wild-type (WT)-hERG and L539fs/47-hERG plasmids were previously constructed at the Department of Cardiovascular Medicine, First Affiliated Hospital of Xi'an Jiaotong University (Xi'an, China); the mutation sequence was obtained from the LQTS 2 family proband. WT or L539fs/47 hERG-pEGFP-C2 plasmids were constructed containing green fluorescent protein (GFP) and the target gene. The L539fs/47-hERG plasmid was sequenced by Sangon Biotech Co., Ltd. (Shanghai, China). The following two primers were used: 5'-AGCGAACCCACA ATGTCACT-3' and 5'-GAGTAGCGATCCAGCTTCCG-3'. The two plasmids were amplified by transformation into Escherichia coli (CB105-02; Tiangen Biotech Co.,Ltd.,Beijing, China), and were extracted using a Roche Genopure Plasmid Maxi kit (Roche Diagnostics GmbH, Mannheim, Germany). The plasmids were sequenced and verified using BLAST [Nucleotide collection (nr/nt) database; https://blast.ncbi.nlm. nih.gov/Blast.cgi] with the WT-hERG cDNA sequence and SeqMan software (version 7.1.0).

Cell culture and model construction. As 293 cells have no endogenous protein expression of hERG, 293 cells were selected for transfection. The 293 cells (American Type Culture Collection, Manassas, VA, USA) were incubated in high-glucose DMEM (HyClone; GE Healthcare Life Sciences, Logan, UT, USA) with $10 \%$ fetal bovine serum (Sijiqing Biological Engineering Materials, Hangzhou, China) at $5 \% \mathrm{CO}_{2}$ and $37^{\circ} \mathrm{C}$. The cells were digested with $0.25 \%$ trypsin (HyClone; GE Healthcare Life Sciences) when they reached $\sim 80 \%$ confluence.

The cells were divided into three groups to establish the different cell models. A total of $4 \mu \mathrm{g}$ of plasmid DNA and $10 \mu \mathrm{l}$ of X-tremeGENE HP DNA transfection reagent (Roche Diagnostics $\mathrm{GmbH}$ ) were incubated in $200 \mu \mathrm{l}$ DMEM for 20 mins prior to being added to the medium. Following incubation in 35-mm dishes (cat. no. 430165; Corning Inc., Corning, NY, USA) at $5 \% \mathrm{CO}_{2}$ and $37^{\circ} \mathrm{C}$ for $12 \mathrm{~h}$ 
(70-90\% confluence), the 293 cells were ready to be transfected. The first group was transfected with $4 \mu \mathrm{g}$ of WT-hERG plasmid to construct the WT cell model. The second group was transfected with $4 \mu \mathrm{g}$ of L539fs/47-hERG plasmid to construct the homozygous mutation cell type. The third group was transfected with $2 \mu \mathrm{g}$ of WT-hERG plasmid and $2 \mu \mathrm{g}$ of L539fs/47-hERG plasmid simultaneously to construct the heterozygous mutation cell model. Following incubation at $5 \% \mathrm{CO}_{2}$ and $37^{\circ} \mathrm{C}$ for 4- $6 \mathrm{~h}$, the culture medium was replaced to minimize damage from the transfection agent. The subsequent experiments were conducted at least $24 \mathrm{~h}$ following transfection, when the hERG channel was amply expressed on the cytomembrane.

Confocal laser scanning microscopy analysis. To verify the distribution of hERG channel proteins in the cell, a fluorescein-labeled hERG plasmid was used together with the pDsRed2-ER plasmid to transfect the 293 cells. The day before transfection, the three groups of cells were subcultured in 35-mm glass bottom dishes (Nest Biotechnology, Wuxi, China) for confocal visualization at a density of $1 \times 10^{5}$ cells per dish. The cells were then transfected with $4 \mu \mathrm{g}$ of WT-hERG-pEGFP-C2, $4 \mu \mathrm{g}$ of L539fs/47-hERG-pEGFP-C2 or $2 \mu \mathrm{g}$ of WT-hERG-pEGFP-C2 and $2 \mu \mathrm{g}$ of L539fs/47-hERGpEGFP-C2. Each plate was also simultaneously transfected with $4 \mu \mathrm{g}$ of pDsRed2-ER plasmid. Following incubation overnight, the cells were observed using a two-channel confocal laser scanning microscope (Nikon C2; Xi'an Jiaotong University, registration no. 21207771).

Treatment with 4-phenyl butyric acid (4-PBA). The chemical chaperone 4-PBA is an ERS inhibitor. 4-PBA (Sigma-Aldrich, Merck KGaA, Darmstadt, Germany) was dissolved in dimethyl sulfoxide (DMSO) and diluted to $1 \mathrm{~mol} / \mathrm{l}$ for storage. The 4-PBA was added to the cell culture medium immediately following transfection. An MTT assay was used to identify the interference concentration of 4-PBA that did not affect cell activity (data not shown). The concentration of $50 \mu \mathrm{mol} / 1$ was selected as the 4-PBA interference concentration.

Hoechst 33342 staining. Hoechst 33342 (Wanlei Bio Co., Ltd., Shanghai, China) staining is a typical cell apoptosis detection method. Sterile coverslips were placed in the bottom of 6-well plates prior to seeding with 293 cells. Following transfection with plasmids and overnight incubation, the culture medium was discarded and the cells were rinsed twice with PBS. The coverslips were covered with $4 \%$ paraformaldehyde for 5 mins and subsequently washed three times with PBS. Hoechst 33342 was then added to the dishes and incubated in the dark for 5 mins. The wells were rinsed twice using PBS prior to observation with a fluorescence microscope (Olympus BX51; Olympus Corporation, Tokyo, Japan). The numbers of apoptotic cells in each group were counted in at least three microscopic visual fields.

Western blot analysis. Following incubation for $24 \mathrm{~h}$, the cells were washed twice with ice-cold PBS. RIPA lysis buffer $(150 \mu 1$, Genshare Biological, Xi'an, China) mixed with $1 \%$ PMSF (Roche Diagnostics, Basel Switzerland) and 2\% phosphatase inhibitor (Genshare Biological) was added to the cells and the homogenate was collected using a cell scraper. The samples were centrifuged at $12,000 \mathrm{x} \mathrm{g}$ and $4^{\circ} \mathrm{C}$ for $30 \mathrm{mins}$ prior to collection of the supernatant whole-cell protein. The protein extracts were quantified using a BCA protein assay kit (Thermo Fisher Scientific, Inc., Waltham, MA, USA) and boiled for 5 mins prior to being applied to SDS-PAGE gels (quantity of protein loaded, $20 \mu \mathrm{g}$ ). The separation gels range from $8-12 \%$ according to the target protein weight. The proteins were separated by electrophoresis at $80 \mathrm{mV}$ for $30 \mathrm{mins}$ and $120 \mathrm{mV}$ for $60-90$ mins. The target protein bands were then cut and set in the transmembrane unit. Following electrophoresis at $200 \mathrm{~mA}$ for $60-80 \mathrm{mins}$, the proteins were transferred onto a PVDF membrane. The membrane was then submerged in blocking buffer (TBST with 5\% milk) at room temperature for $1 \mathrm{~h}$, followed by incubation in diluted primary antibody at $4^{\circ} \mathrm{C}$ overnight. The membrane was subsequently incubated in diluted HRP-labeled secondary antibody for $1 \mathrm{~h}$ at room temperature. The bands were detected using a chemiluminescence detector (Bio-Rad ChemiDoc XRS, Bio-Rad Laboratories, Inc., Hercules, CA, USA) and were analyzed using Quantity One software (version, 4.6.7).

The primary antibodies were as follows: hERG (sc-377388; 1:500; Santa Cruz Biotechnology, Inc., Dallas, TX, USA), GRP78 (cat. no. WL0781; 1:1,000; Wanleibio Co., Ltd., Shanghai, China), phosphorylated (p-)PERK (cat. no. bs-23340R; 1:500; Bioss, Beijing, China), p-eIF2 $\alpha$ (cat. no. 3398; 1:1,000; Cell Signaling Technology, Inc., Danvers, MA, USA), CHOP (cat. no. WL00880; 1:1,000), Bax (cat. no. WL01637; 1:500), Bcl-2 (cat. no. WL01556; 1:500), cleaved-caspase-3 (cat. no. WL01857; 1:500; all Wanleibio Co., Ltd.), caspase-12 (cat. no. 2202; 1:1,000) and GAPDH (cat. no. sc-25778; 1:1,000; both Santa Cruz Biotechnology, Inc.). The secondary antibodies were goat anti-rabbit antibody (cat. no. WL023a; 1:5,000; Wanleibio Co, Ltd.) and rabbit anti-mouse antibody (cat. no. 31430; 1:10,000; Thermo Fisher Scientific, Inc.).

Statistical analysis. All data are presented as the mean \pm standard deviation and were analyzed using SPSS version 21.0 software (IBM Corp., Armonk, NY, USA). One-way analysis of variance was used to compare the means of more than two groups when the data were distributed according to normality and homogeneity of variance. Pairwise comparisons were conducted using the LSD test. $\mathrm{P}<0.05$ was considered to indicate a statistically significant difference.

\section{Results}

Cell model construction and verification. The results of DNA sequencing showed that the L539fs/47-hERG plasmid contained a mutated hERG segment characterized by the absence of a 19-bp segment at the 1619-1637 site (CCG TACTCTGAGTAGCGAT) and an $\mathrm{A} \rightarrow \mathrm{G}$ mutation at 1692 site (Fig. 1A). Protein translation ceased at the 4th transmembrane section of the hERG channel protein (Fig. 1B).

At $24 \mathrm{~h}$ post-transfection, the hERG protein was sufficiently expressed to be detectable by western blot analysis. A mouse primary antibody targeted the hERG $\mathrm{N}$-terminus. This antibody also detected the truncated L539fs/47-hERG protein. The WT-hERG showed two bands, one at $135 \mathrm{kDa}$ and another at 
A

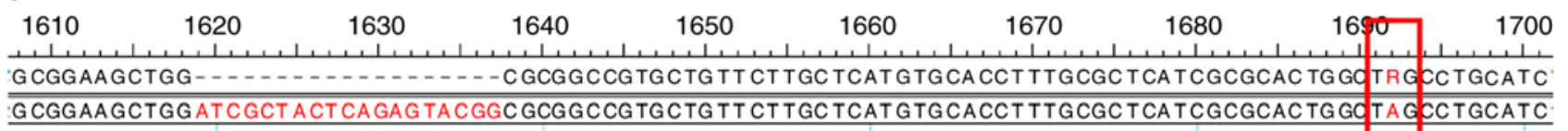

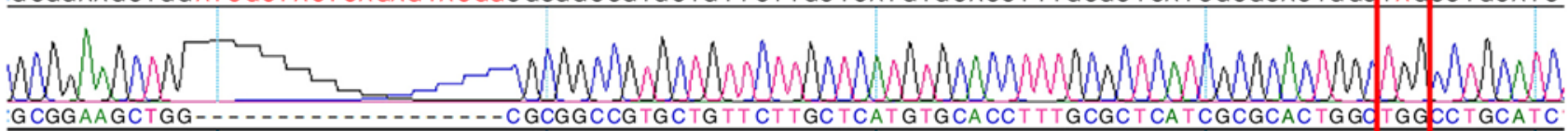
GCGGAAGCTGG-............... Wh: Why

$\uparrow$

$1619 \rightarrow 163719 \mathrm{bps}$

deletion $\uparrow$

$1692 A \rightarrow G$

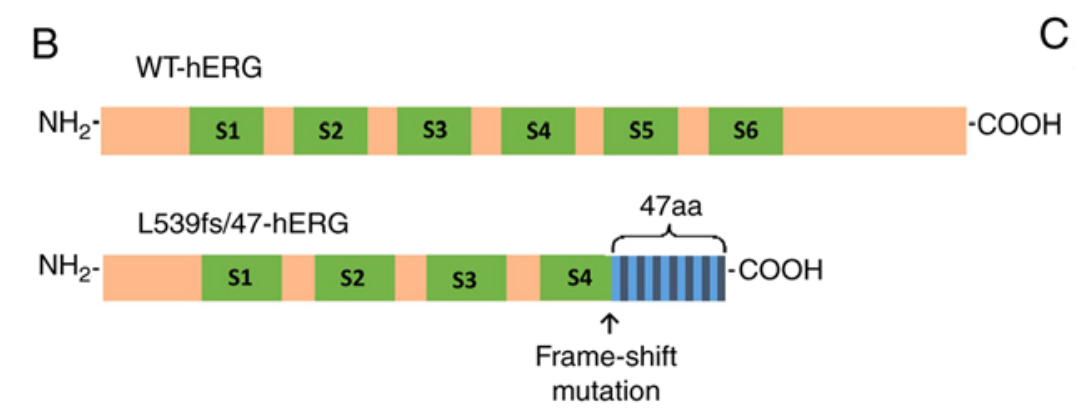

C

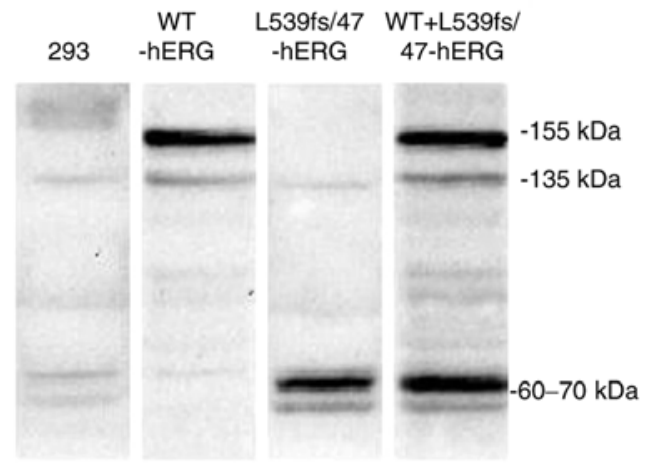

Figure 1. Structure of L539fs/47-hERG. (A) Sequencing result of L539fs/47-hERG and BLAST results for the WT-hERG sequence. (B) Diagram of hERG and the L539fs/47-hERG truncated protein structure. Following transmembrane section S4, the protein is disordered and truncated. (C) Mutated hERG protein is truncated to $\sim 60-70 \mathrm{kDa}$. hERG, human ether-à-go-go-related gene; WT, wild-type.

$155 \mathrm{kDa}$, whichrepresentedthenon-glycosylatedandglycosylated protein, respectively. The L539fs/47-hERG homozygous mutant protein only exhibited a band at 60-70 kDa, which was attributed to protein truncation. The heterozygous mutant cell type had the 135 and $155 \mathrm{kDa}$ bands but also the truncated protein band at 60-70 kDa (Fig. 1C).

The distribution of the hERG channel protein was detected by confocal laser scanning microscopy. The red florescence label calreticulin marked the endoplasmic reticulum, and the green florescence indicated the location of the hERG protein. In the WT-hERG cells, the green florescence from the WT-hERG protein was located predominantly at the cell outer membrane (Fig. 2A-C). The middle row in Fig. 2 indicates that the L539fs/47-hERG homozygous mutant cells tended to have channel proteins mainly located in the inner cell structure (Fig. 2D-F), particularly in the ER, which is shown as yellow florescence in the merged image. The bottom row in Fig. 2 shows the WT-L539fs/47-hERG heterozygous mutation. The channel protein was present in the ER and at the cell membrane, and the merged image shows yellow fluorescence inside the cell and green fluorescence around the cell (Fig. 2G-I). The results demonstrate that the mutated L539fs/47-hERG protein was recognized and retained in the ER and was not expressed on the cell membrane. These results confirm our previous results regarding L539fs/47-hERG (20).

L539fs/47-hERG triggers ERS. GRP78 is a chaperone anchored in the ER that can recognize unfolded proteins and assist in their complete folding or lead them to degradation. Therefore, it is the most representative marker of ERS activation. Increasing the expression of GRP78 under ERS conditions tends to relieve the ER burden. The L539fs/47-hERG protein has been demonstrated to be a truncated protein that is retained in the ER. To ascertain whether the abundant misfolded protein triggered ERS, whole-cell protein was extracted from the WT-hERG, L539fs/47-hERG and WT-L539fs/47-hERG cells to evaluate the expression levels of GRP78 by western blot analysis. These samples were compared with extracted whole-cell protein of the three groups in which the ERS inhibitor 4-PBA was added. The 4-PBA concentration was $50 \mu \mathrm{mol} / 1$ and was determined according to prior experiments.

The three groups of cells showed significant differences in expression levels of GRP78. The expression of GRP78 was significantly higher in the L539fs/47 cells compared with that in the WT cells (Fig. 3A and B). Compared with the WT cells, the L539fs/47 cells showed significantly higher expression of GRP78 ( $\mathrm{P}=0.004)$, whereas expression in the WT-L539fs/47 cells was similar to that in the WT cells, showing no significant difference in the expression of GRP78 ( $\mathrm{P}=0.120)$. The ERS inhibitor 4-PBA significantly reduced the expression of GRP78 in the L539fs/47-hERG cells $(\mathrm{P}=0.039)$, showing that the mutation-induced ERS was reversed by 4-PBA. Furthermore, following 4-PBA treatment, the WT-L539fs/47-hERG cells exhibited a small decrease in expression of GRP78 ( $\mathrm{P}=0.573)$, whereas the WT-hERG cells exhibited higher expression of GRP78 ( $\mathrm{P}=0.041)$, indicating 


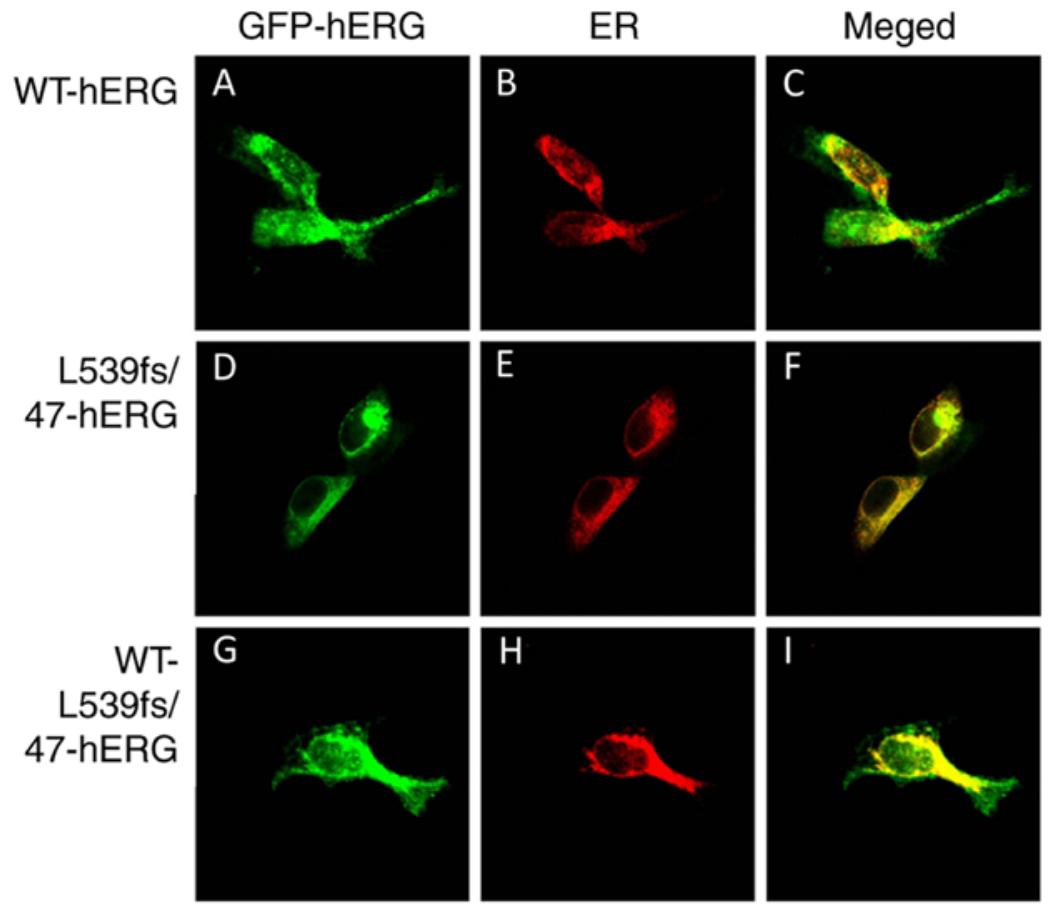

Figure 2. Confocal laser scanning of the hERG protein distribution under x600 magnification. The red fluorescence represents the ER structure; the green florescence represents the location of hERG protein. The yellow florescence in the merged images is the overlapping color of green and red florescence, which indicates the hERG protein is retained in the ER. Images show protein distribution by (A) GFP, (B) ER and (C) merged fluorescence in WT-hERG cells; (D) GFP, (E) ER and (F) merged fluorescence in L539fs/47-hERG cells; and (G) GFP, (H) ER and (I) merged fluorescence in WT-L539fs/47-hERG cells. hERG, human ether-à-go-go-related gene; WT, wild-type; GFP, green fluorescent protein; ER, endoplasmic reticulum.

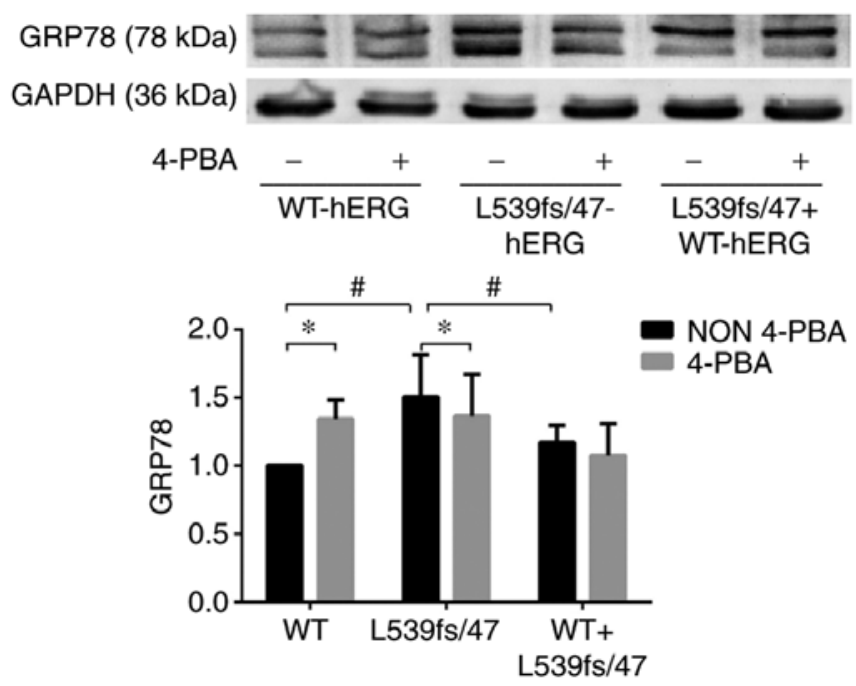

Figure 3. Expression of GRP78 detected by western blot analysis. The experiment was repeated three times. " $\mathrm{P}<0.05$ between the three model cell groups; ${ }^{*} \mathrm{P}<0.05$ between the two subgroups treated with or without 4 -PBA intervention. $\mathrm{P}<0.05$ was considered to indicate a statistically significant difference. hERG, human ether-à-go-go-related gene; GRP78, glucose related protein 78; WT, wild-type; 4PBA, 4-phenyl butyric acid.

that ERS was not activated in these two cells. Therefore, retention of the homozygous hERG mutant protein in the ER was able to trigger ERS.

L539fs/47-hERG causes cell apoptosis. It is well known that patients with LQTS exhibit classical cardiomyocyte electrical activity disorder. In addition, multiple studies have reported that the hERG channel mutation can induce cell apoptosis, not only in heart biopsies from patients, but also in studies in vitro $(8,9,10)$. Therefore, it was hypothesized that the hERG missense mutation, L539fs/47-hERG, also causes cell apoptosis. To verify this, 293 cells were divided into three groups: WT-hERG, L539fs/47-hERG and WT-L539fs/47-hERG. Each of these groups was then divided into two sub-groups, with or without 4-PBA treatment. Cell apoptosis was then detected by Hoechst 33342 apoptosis staining.

The three groups of cells were stained with Hoechst 33342 , in which bright blue florescence indicates chromatin gathering and karyopyknosis in the process of cell apoptosis. As shown in the upper row of Fig. 4A, the apoptotic rates of the three groups were significantly different $(2.50 \pm 1.26$, $11.78 \pm 1.69$ and $4.41 \pm 0.30 \%$ respectively, $\mathrm{P}<0.01$; Fig. $4 \mathrm{~B}$ ). The L539fs/47-hERG cells exhibited a greater extent of apoptosis (red arrow) compared with the WT-hERG cells $(\mathrm{P}<0.01)$. The WT-L539fs/47-hERG heterozygous mutant cells exhibited staining indicating sporadic apoptosis compared with the other two groups (Fig. 4B). These results indicated that the L539fs/47-hERG homozygous mutation led to cell apoptosis.

ERS inhibitor 4-PBA can reverse the apoptosis induced by mutation. Long-term or intense ERS promotes apoptosis. The results showed that the channel gene mutation L539fs/47-hERG triggered ERS, which subsequently induced cell apoptosis. To further verify this, the ERS inhibitor 4-PBA was introduced to interrupt ERS in the L539fs/47-hERG cells and observe the state of apoptosis. The results, shown in the bottom row 


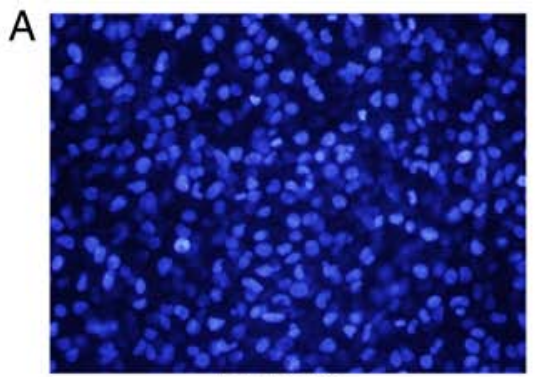

WT-hERG

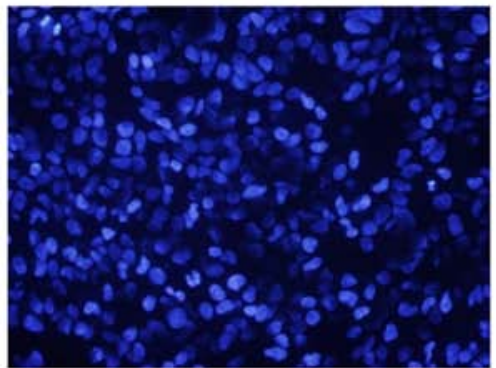

WT-hERG

+4-PBA

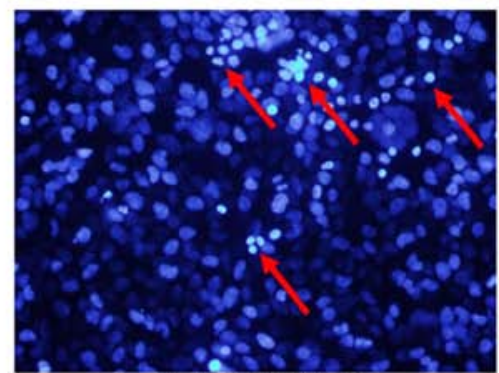

L539fs/47-hERG

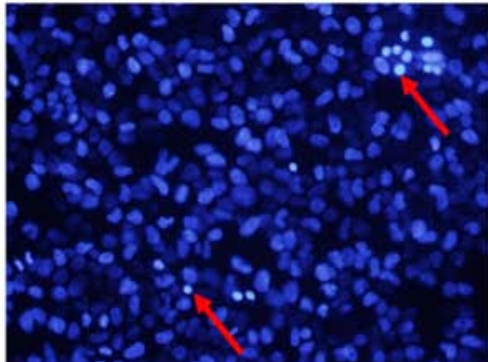

L539fs/47-hERG +4-PBA

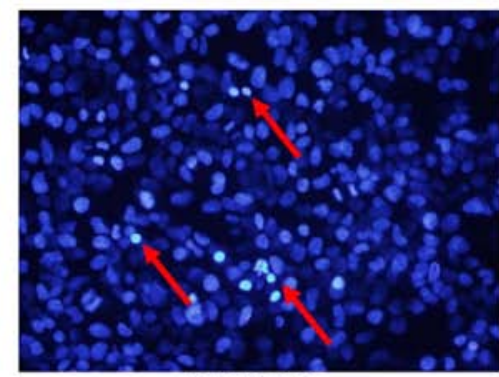

WT-hERG+ L539fs/47-hERG

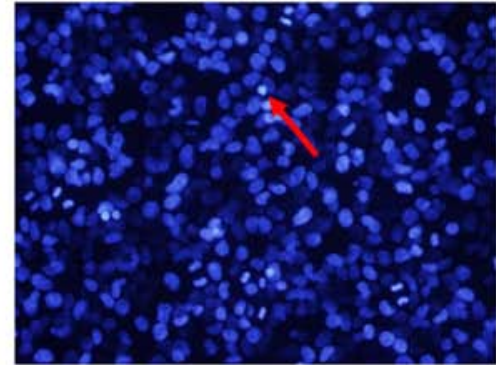

Wt-hERG+ L539fs/47-hERG+4-PBA

B

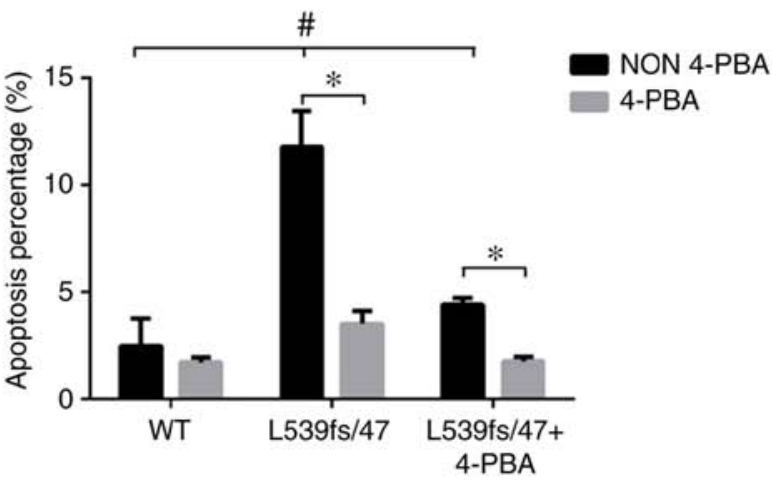

Figure 4. Hoechst 33342 staining of model cell apoptosis (x200 magnification). The experiment was repeated three times. (A) Red arrows indicate apoptotic cells. The images represent the three cell models transfected with plasmids WT-hERG, L539fs/47-hERG or WT-L539fs/47-hERG, with or without 50 $\mu$ mol/1 4-PBA intervention. (B) Percentage of apoptotic cells in each group. " $\mathrm{P}<0.05$ between the three model cell groups; ${ }^{*} \mathrm{P}<0.05$ between the two subgroups treated with or without 4-PBA intervention. $\mathrm{P}<0.05$ was considered to indicate a statistically significant difference. hERG, human ether-à-go-go-related gene; WT, wild-type; 4PBA, 4-phenyl butyric acid.

of Fig. 4A, indicate that treatment with $50 \mu \mathrm{mol} / 1$ 4-PBA notably reduced apoptosis in the homozygous and heterozygous mutated cells $(3.53 \pm 0.59$ and $1.79 \pm 0.22 \%, \mathrm{P}=0.014$ and 0.003 , respectively) but had no effect on WT-hERG cells $(1.75 \pm 0.22 \%, \mathrm{P}=0.437)$ (Fig. 4B). These findings revealed that the L539fs/47-hERG-induced apoptosis was a result of ERS activation, and that ERS inhibition by 4-PBA prevented apoptosis in the mutated cells.

Examination of the mechanisms of L539fs/47-hERG-induced apoptosis. The results demonstrated that L539fs/47-hERG can cause cell apoptosis by inducing the activation of ERS. Therefore, the present study focused on elucidating which ERS molecular pathways involving the hERG mutation were active in inducing the progression of apoptosis. The 293 cells were divided into three groups and transfected with hERG plasmids. Pathway-associated protein expression was detected by western blot analysis. The results are shown in Fig. 5A and B. Accordingly, the PERK-eIF $2 \alpha$-CHOP pathway was activated in L539fs/47 cells.
Following transfection, the activation of PERK to p-PERK was significantly increased in the L539fs/47 cells compared with the WT and heterozygous cells $(\mathrm{P}=0.007$ and 0.014 , respectively). The downstream molecule of p-PERK, eIF $2 \alpha$, is an important regulator mediating apoptosis in ERS. The expression of eIF $2 \alpha$ was elevated in the homozygous mutant L539fs/47 cells ( $\mathrm{P}=0.005$ and 0.037 compared with WT and WT-L539fs/47, respectively). The PERK-eIF2 $\alpha$-CHOP pathway is essential in upregulating CHOP-mediated cell apoptosis. Significantly higher levels of CHOP were observed in the L539fs/47 cells $(\mathrm{P}=0.005)$, with only marginal elevation observed in the WT-L539fs/47 cells $(\mathrm{P}=0.089)$, compared with the WT cells. Bax/Bcl-2 regulation was also indicated to be involved in L539fs/47-induced cell apoptosis. The L539fs/47 cells exhibited a significantly higher $\mathrm{Bax} / \mathrm{Bcl}-2$ ratio compared with that in the WT cells $(\mathrm{P}=0.009)$. However, the expression levels of p-PERK, eIF $2 \alpha$, CHOP and $\mathrm{Bax} / \mathrm{Bcl}-2$ in the WT-L539fs/47 heterozygous mutant cells were not significantly elevated compared with those in the WT cells $(\mathrm{P}=0.569,0.147,0.059$ and 0.058 , respectively). 
A

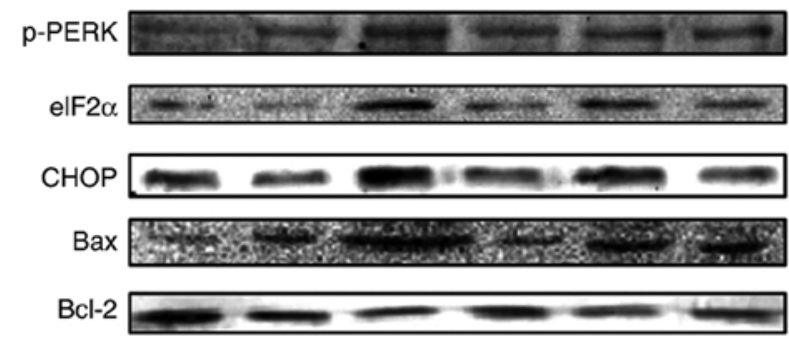

Caspase-12 20
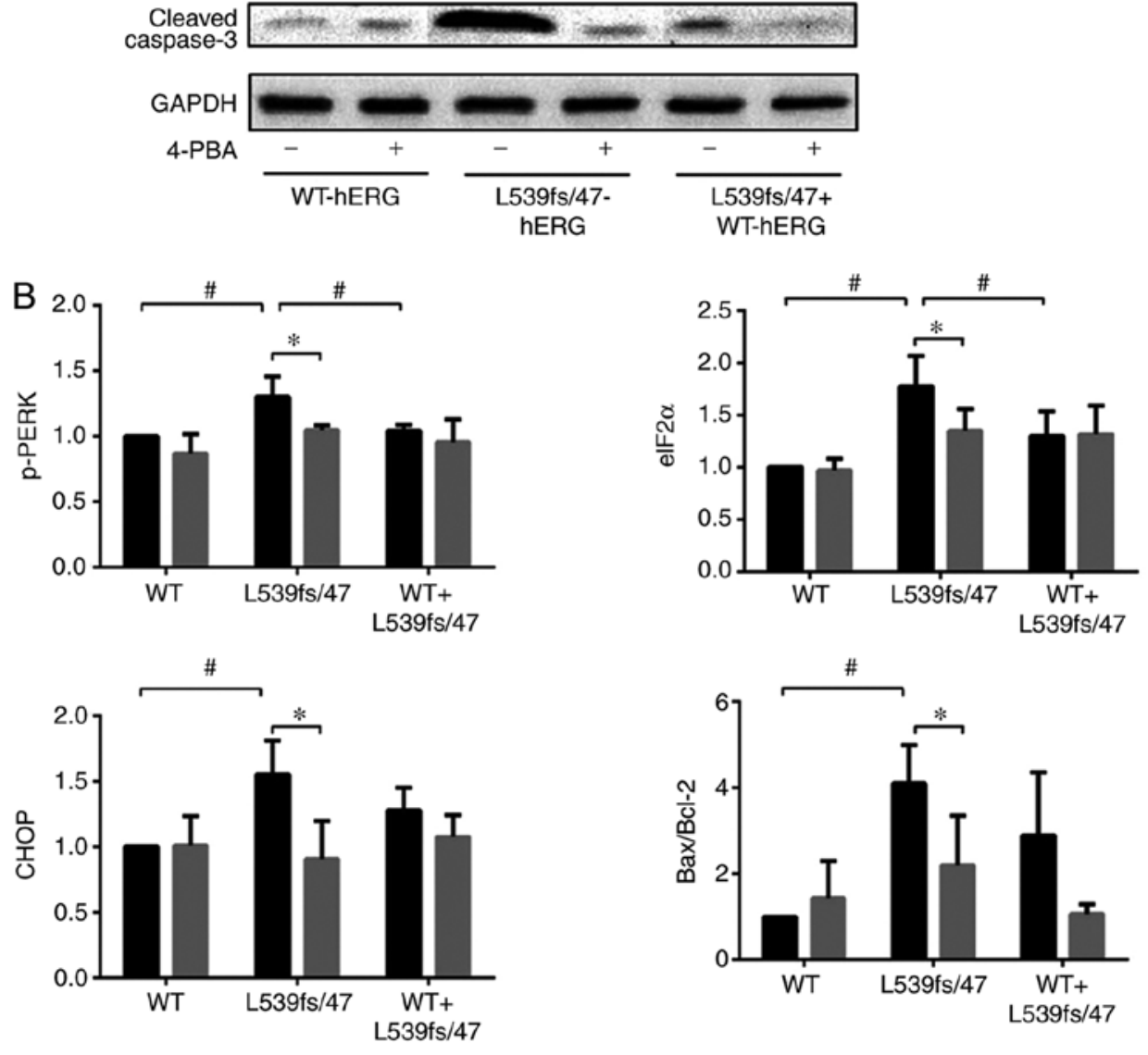

NON 4-PBA

4-PBA
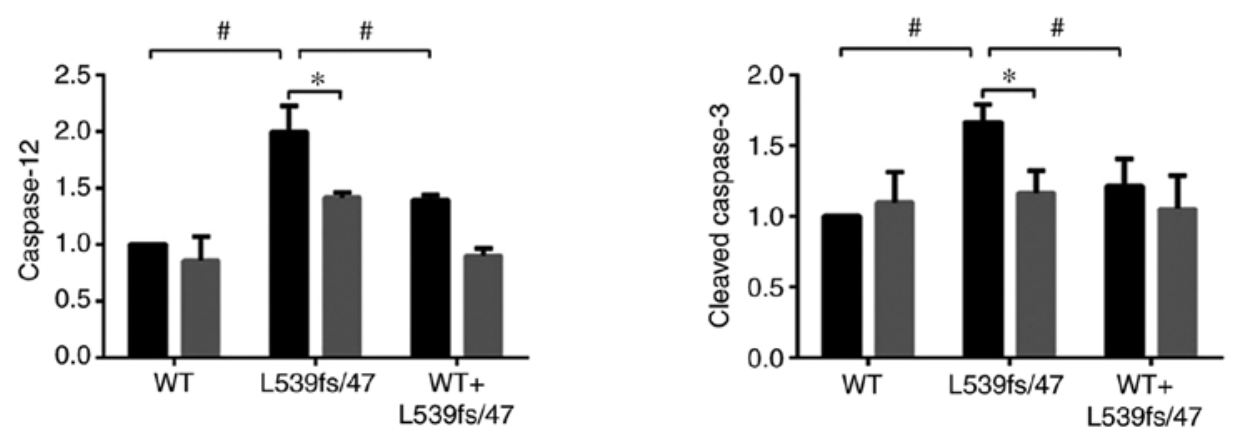

Figure 5. Western blot analysis results for ERS-associated proteins. (A) The representative blotting images and (B) quantified data demonstrate elevated p-PERK, eIF2a, CHOP, Bax/Bcl-2, Caspase-12 and cleaved Caspase-3 level in L539fs/47-hERG cells, which can be reversed by 4-PBA. Each experiment was repeated at least three times. ${ }^{*} \mathrm{P}<0.05$ between the three model cell groups; ${ }^{*} \mathrm{P}<0.05$ between two subgroups treated with or without 4 -PBA intervention. $\mathrm{P}<0.05$ was considered to indicate a statistically significant difference. ERS, endoplasmic reticulum stress; hERG, human ether-à-go-go-related gene; WT, wild-type; 4PBA, 4-phenyl butyric acid; p-PERK, phosphorylated protein kinase R-like endoplasmic reticulum kinase; eIF2a; eukaryotic translation-initiation factor-2 $\alpha$; CHOP, C/EBP homologous protein; Bcl2, B-cell lymphoma 2; Bax, Bcl-2-associated X protein.

As shown in Fig. 5A and B, the results showed increased expression of caspase-12 in the L539fs/47-hERG-transfected cells compared with that in the WT $(\mathrm{P}<0.05)$ and WT-L539fs/47 $(\mathrm{P}<0.05)$ cells. The expression of caspase-12 in the heterozygous mutant cells was also higher compared with that in the WT cells $(\mathrm{P}=0.013)$. The active form of caspase-3, cleaved-caspase-3, was significantly increased in the L539fs/47 mutation group compared with that in the other two groups 
$(\mathrm{P}=0.001$ and 0.006$)$. However, no significant difference in expression was observed between the WT-L539fs/47 cells and WT cells $(\mathrm{P}=0.099)$.

Treatment with 4-PBA decreased the PERK-eIF2 $\alpha$-CHOP pathway, Bax/Bcl-2 ratio, and expression levels of caspase-12 and cleaved-caspase-3 in the L539fs/47 homozygous mutant cells (all $\mathrm{P}<0.05$; Fig. $5 \mathrm{~A}$ and $\mathrm{B}$ ) but not in the WT or WT-L539fs/47 cells. These results support the results of GRP78 described above and suggest that cell apoptosis mediated by different activated pathways in L539fs/47 cells is the result of ERS.

\section{Discussion}

Historically, LQTS has been defined by abnormalities in electrical activity due to its unusual ECG appearance. As early recognition and intervention has become more effective, patients with LQTS have a longer average life expectancy; therefore, structural abnormalities that were hidden by early onset sudden mortality are now being revealed to clinicians and researchers. As mentioned above, several heart structural abnormalities are associated with LQTS gene mutations, however, these cases have received less attention. Almost all previous studies and clinical guidelines have focused on abnormal ECG activities and not on structural defects; a limited number of studies have investigated the mechanisms of LQTS-induced structural abnormalities. Cell apoptosis is an important natural procedure in the development of the cardiovascular system (21). However, extensive apoptosis in the fetal heart can induce heart defects. In the mature heart, apoptosis is an initiation factor of fibrosis, ventricle remodeling or contraction abnormality $(22,23)$. A series of studies have shown marked apoptosis in cells with LQTS gene mutations using human heart biopsies and in vivo and in vitro methods. In other areas, including cancer research, the hERG channel has been found to be an important component in the regulation of apoptosis. In colon carcinoma, esophageal squamous cell carcinoma, leukemic cells, endometrial cancer cells and cervical squamous cell carcinoma cells, hERG has a higher expression level and exhibits anti-apoptotic behaviors. The application of an hERG inhibitor can reverse cancer cell apoptosis (24).

LQTS 2 is the most common type of LQTS in China, and it accounts for $54.5 \%$ of all congenital LQTS cases (25). Our previous study examined a family diagnosed with the L539fs/47-hERG mutation. The proband was a 20 -year-old female who experienced her first syncope upon waking. The proband's ECGs showed a QTc of $0.60 \mathrm{sec}$ with paroxysmal polytypic ventricular tachycardia. DNA sequencing of the family members revealed that the proband's father and one brother had the KCNH2 gene mutation L539fs/47-hERG. L539fs/47 is a complex mutation, as is previously described. According to the results of our previous study of mutated hERG channel function, the L539fs/47-hERG protein does not have normal channel function (20). The results of the present study using confocal laser microscopy verified the results of the previous study, indicating that the mutated protein can be recognized by the ER and be retained in it.

The in vitro experiments performed in the present study also identified that the homozygous mutated L539fs/47-hERG protein can lead to cell apoptosis. Similar trends were identified on investigating the molecular pathway; elevated expression levels of GRP78 were found in L539fs/47-hERG homozygous cells. It was also found that the PERK-eIF2 $\alpha$-CHOP pathway was involved in L539fs/47-hERG-induced ERS-mediated cell apoptosis. Heterozygous mutations of L539fs/47 did not provoke ERS to induce apoptosis. The same results were also identified in the analyses of $\mathrm{Bax} / \mathrm{Bcl}-2$ and caspase-12 (Fig. 6). The mutation caused apoptosis and molecular changes, which were reversed by intervention with the ERS inhibitor 4-PBA. These results indicate that hERG homozygous mutation-induced cell apoptosis may be the underlying mechanism of LQTS 2 structural abnormalities and that ERS, which is provoked by the mutated protein retained in the ER lumen, is the mechanism of apoptosis. In clinical practice, homozygous mutations of hERG always cause severe symptoms and can lead to mortality during the embryonic period; however, individuals with heterozygous mutations can survive and can be asymptomatic. For this reason, the majority of patients with LQTS 2 carry a heterozygous mutation. The results of the present study showed that the L539fs/47-hERG homozygous mutation resulted in more marked effects on cell apoptosis and molecular biology, with similar manifestations to LQTS 2 . This is likely to explain why the cardiovascular structural abnormalities of patients with LQTS remained undetected for so long. In addition, as shown in Fig. 3, 4-PBA reduced the expression of GRP78 in the L539fs/47-hERG and WT-L539fs/47-hERG cells, but resulted in higher expression of GRP78 $(\mathrm{P}=0.041)$ in the WT-hERG cells. This result indicated that ERS was not activated in WT-hERG cells. The elevation of GRP78 may be the result of other factors, including the 4-PBA solvent DMSO or other random errors.

To the best of our knowledge, the present study is the first to demonstrate the role of the ERS pathway PERK-eIF2 $\alpha$-CHOP in LQTS 2-induced cell apoptosis. An additional molecule, ATF6, can also be activated in LQTS mutation-induced ERS. In a study by Wang et al, the hERG mutations G572R and E637K activated the expression of ATF6 and induced mutated protein degradation through the ubiquitin-mediated proteasome pathway (19). Keller et al found that NF- $\mathrm{KB}$ signaling is activated by the I539R-hERG mutation (18). Whether the two ERS molecules ATF6 and IRE1, or other pathways, such as NF- $\kappa \mathrm{B}$, are involved in L539fs/47-hERG-induced cell apoptosis requires further investigation.

The L539fs/47-hERG mutation is a functional deletion mutation that disables the potassium ion channel function of the protein; therefore, the balance of ions, such as $\mathrm{Ca}^{2+}$ and $\mathrm{Na}^{+}$, between the cytoplasm and the extracellular matrix is significantly altered by decreasing the $\mathrm{K}^{+}$outflow. This change may trigger cell apoptosis through multiple mechanisms. Under ERS conditions, multiple factors are involved in the regulation of $\mathrm{ER} \mathrm{Ca}^{2+}$ release; for example, $\mathrm{CHOP}$ and $\mathrm{Bcl}-2$ lead to the accumulation of $\mathrm{Ca}^{2+}$ in the cytoplasm $(26,27)$. High $\mathrm{Ca}^{2+}$ concentrations can cause cell apoptosis via the activation of calpain. Calpain is a widely distributed protease in mammalian cells. It can be activated by $\mathrm{Ca}^{2+}$ and subsequently induce caspase and non-caspase cell apoptotic pathways. An increased calpain has been found in apoptotic L539fs/47-hERG cells (28). Additional pathways may also be mechanisms of 


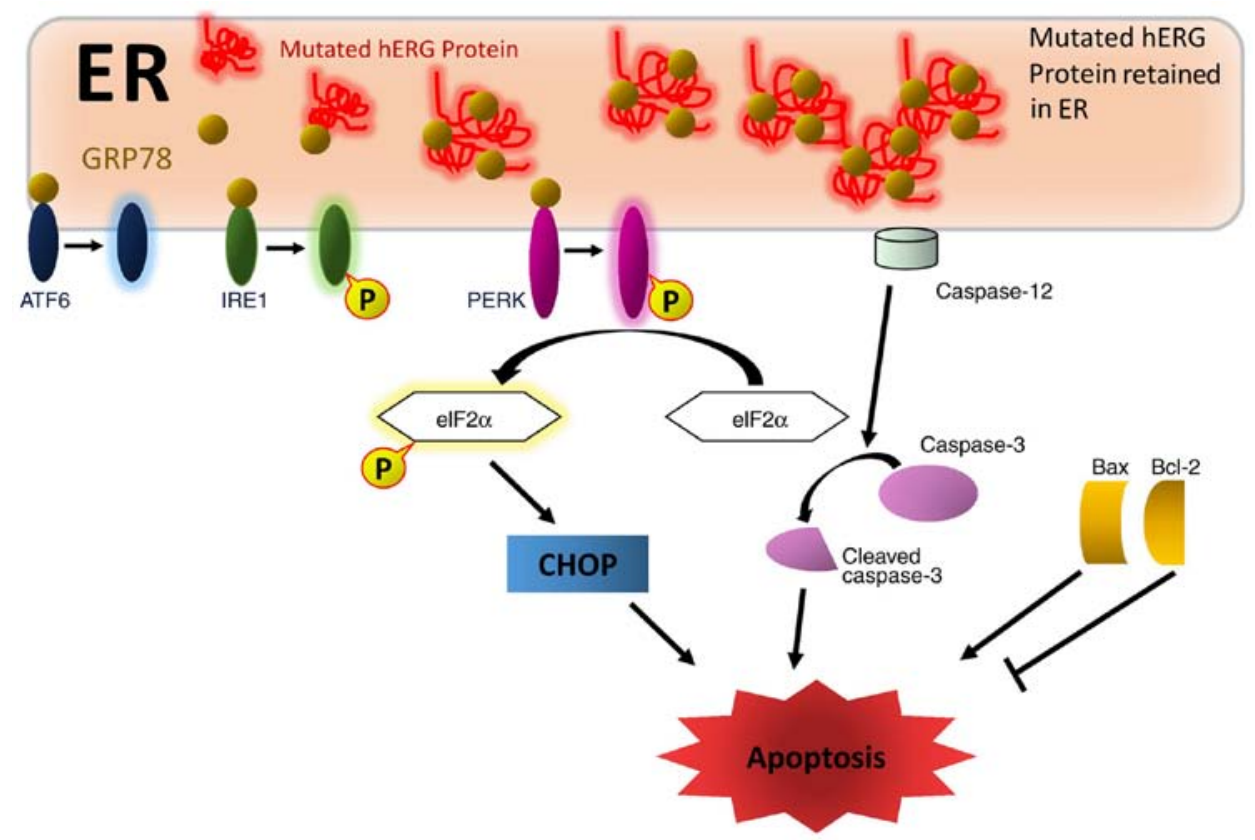

Figure 6. Proposed mechanisms of mutated hERG-induced cell apoptosis. Mutated L539fs/47-hERG protein retained in the ER lumen provokes ERS. Elevated expression levels of GRP78 activate the PERK-eIF2 $\alpha$-CHOP pathway, which is important in the mechanism of L539fs/47-hERG-induced ERS-mediated cell apoptosis. ER-specific caspase-12 is involved in the apoptotic mechanisms caused by the hERG mutation through cleaving and activating caspase-3. The pair of apoptosis factors Bax/Bcl-2 also regulate L539fs/47-hERG-induced cell apoptosis. hERG, human ether-à-go-go-related gene; WT, wild-type; 4PBA, 4-phenyl butyric acid; ER, endoplasmic reticulum; ERS, ER stress; ATF6, activating transcription factor 6; IRE1, inositol-requiring enzyme 1; p-PERK, phosphorylated protein kinase R-like endoplasmic reticulum kinase; eIF2a; eukaryotic translation-initiation factor-2 $\alpha$; $\mathrm{CHOP}, \mathrm{C} / \mathrm{EBP}$ homologous protein; Bcl2, B-cell lymphoma 2; Bax, Bcl-2-associated X protein.

LQTS 2-related gene mutations that cause cell apoptosis, including calcium overload or oxidative stress.

The results of the present study were obtained based on in vitro experiments. Under in vivo conditions, multiple ion channels and several biological factors are involved in the process of LQTS-induced cell apoptosis. Furthermore, the protein expression of $\mathrm{hERG}$ is differential in different regions of the heart, which may evoke ER stress to varying degrees. The variability of cardiomyocyte apoptosis is a potential mechanism in the pathological process of LQTS. Therefore, to identify other mechanisms involved in hERG mutation-induced cell apoptosis, an LQTS type 2 transgenic animal is to be used in future investigations.

In conclusion, the present study is the first, to the best of our knowledge, to investigate the mechanisms of cardiomyocyte apoptosis caused by hERG channel mutations. It was found that L539fs/47-hERG mutated protein accumulates in the ER and induces ERS, and ERS-associated cell apoptosis is the mechanism by which the mutations are able to cause apoptosis. These mechanisms of LQTS heart structural abnormalities may be future targets of intervention in the treatment of patients with LQTS. The results also provide novel strategies for the investigation of heart diseases in children, including congenital heart disease, fetal mortality and cardiomyopathies.

\section{Acknowledgements}

The authors would like to thank Dr G. Michael Vincent (LDS Hospital, Salt Lake City, UT, USA) and Dr Blake D. Anson (University of Wisconsin, Madison, WI, USA) for providing the WT-hERG plasmid, and Dr Aifeng Zhang for constructing the L539fs/47-hERG plasmid.

\section{Funding}

The present study was supported by the National Natural Science Foundation of China (grant no. 81270236).

\section{Availability of data and materials}

The datasets used and/or analyzed during the present study are available from the corresponding author on reasonable request.

\section{Authors' contributions}

SM and CS designed the experiments; SM and YZ performed the experiments; $\mathrm{SM}$ and $\mathrm{MC}$ analyzed the experimental data. SM, YZ and CS wrote the manuscript. All authors read and approved the final manuscript.

\section{Ethics approval and consent to participate}

Not applicable.

\section{Patient consent for publication}

Not applicable.

\section{Competing interests}

The authors declare that they have no competing interests. 


\section{References}

1. Ackerman MJ, Priori SG, Willems S, Berul C, Brugada R, Calkins H, Camm AJ, Ellinor PT, Gollob M, Hamilton R, et al: HRS/EHRA expert consensus statement on the state of genetic testing for the channelopathies and cardiomyopathies: This document was developed as a partnership between the Heart Rhythm Society (HRS) and the european Heart Rhythm association (EHRA). Europace 13: 1077-1109, 2011.

2. John RM, Tedrow UB, Koplan BA, Albert CM, Epstein LM, Sweeney MO, Miller AL, Michaud GF and Stevenson WG: Ventricular arrhythmias and sudden cardiac death. Lancet 380 : $1520-1529,2012$.

3. Nador F, Beria G, De Ferrari GM, Stramba-Badiale M, Locati EH, Lotto A and Schwartz PJ: Unsuspected echocardiographic abnormality in the long QT syndrome. Diagnostic, prognostic, and pathogenetic implications. Circulation 84: 1530-1542, 1991.

4. Haugaa KH, Amlie JP, Berge KE, Leren TP, Smiseth OA and Edvardsen T: Transmural differences in myocardial contraction in long-QT syndrome: Mechanical consequences of ion channel dysfunction. Circulation 122: 1355-1363, 2010.

5. Zaklyazminskaya E and Dzemeshkevich S: The role of mutations in the SCN5A gene in cardiomyopathies. Biochim Biophys Acta 1863: 1799-1805, 2016.

6. Walls J, Sanatani S and Hamilton R: Post-hoc diagnosis of congenital long QT syndrome in patients with tetralogy of Fallot. Pediatr Cardiol 26: 107-110, 2005.

7. Murugan SJ, Parsons JM and Bennett C: A case of long QT syndrome associated with familial occurrence of persistent patency of the arterial duct. Cardiol Young 15: 309-311, 2005.

8. James TN, Terasaki F, Pavlovich ER and Vikhert AM: Apoptosis and pleomorphic micromitochondriosis in the sinus nodes surgically excised from five patients with the long QT syndrome. J Lab Clin Med 122: 309-323, 1993.

9. Zhang T, Yong SL, Drinko JK, Popović ZB, Shryock JC, Belardinelli L and Wang QK: LQTS mutation N1325S in cardiac sodium channel gene SCN5A causes cardiomyocyte apoptosis, cardiac fibrosis and contractile dysfunction in mice. Int J Cardiol 147: 239-245, 2011

10. Teng GQ, Zhao X, Lees-Miller JP, Quinn FR, Li P, Rancourt DE, London B, Cross JC and Duff HJ: Homozygous missense N629D hERG (KCNH2) potassium channel mutation causes developmental defects in the right ventricle and its outflow tract and embryonic lethality. Circ Res 103: 1483-1491, 2008.

11. Kim I, Xu W and Reed JC: Cell death and endoplasmic reticulum stress: Disease relevance and therapeutic opportunities. Nat Rev Drug Discov 7: 1013-1030, 2008.

12. Wang $\mathrm{S}$ and Kaufman RJ: The impact of the unfolded protein response on human disease. J Cell Biol 197: 857-867, 2012.

13. Oltvai ZN, Milliman CL and Korsmeyer SJ: Bcl-2 heterodimerizes in vivo with a conserved homolog, Bax, that accelerates programmed cell death. Cell 74: 609-619, 1993.

14. Xu C, Bailly-Maitre B and Reed JC: Endoplasmic reticulum stress: Cell life and death decisions. J Clin Invest 115: 2656-2664, 2005.

15. Nakagawa T, Zhu H, Morishima N, Li E, Xu J, Yankner BA and Yuan J: Caspase-12 mediates endoplasmic-reticulum-specific apoptosis and cytotoxicity by amyloid-beta. Nature 403: 98-103, 2000 .
16. Budihardjo I, Oliver H,Lutter M, Luo X and Wang X: Biochemical pathways of caspase activation during apoptosis. Annu Rev Cell Dev Biol 15: 269-290, 1999.

17. Bohnen MS, Peng G, Robey SH, Terrenoire C, Iyer V, Sampson KJ and Kass RS: Molecular pathophysiology of congenital long QT syndrome. Physiol Rev 97: 89-134, 2017.

18. Keller SH, Platoshyn O and Yuan JX: Long QT syndrome-associated I593R mutation in HERG potassium channel activates ER stress pathways. Cell Biochem Biophys 43: 365-377, 2005.

19. Wang Y, Huang X, Zhou J, Yang X, Li D, Mao H, Sun HH, Liu N and Lian J: Trafficking-deficient G572R-hERG and E637K-hERG activate stress and clearance pathways in endoplasmic reticulum. PLoS One 7: e29885, 2012.

20. Zhang A, Sun C, Zhang L, Lv Y, Xue X, Li G, Cui C and Yan GX: L539 fs/47, a truncated mutation of human ether-a-go-go-related gene (hERG), decreases hERG ion channel currents in HEK 293 cells. Clin Exp Pharmacol Physiol 40: 28-36, 2013.

21. Abdelwahid E, Pelliniemi LJ and Jokinen E: Cell death and differentiation in the development of the endocardial cushion of the embryonic heart. Microsc Res Tech 58: 395-403, 2002.

22. Travers JG, Kamal FA, Robbins J, Yutzey KE and Blaxall BC: Cardiac fibrosis: The fibroblast awakens. Circ Res 118: 1021-1040, 2016.

23. Liu X, Kwak D, Lu Z, Xu X, Fassett J, Wang H, Wei Y, Cavener DR, Hu X, Hall J, et al: Endoplasmic reticulum stress sensor protein kinase R-like endoplasmic reticulum kinase (PERK) protects against pressure overload-induced heart failure and lung remodeling. Hypertension 64: 738-744, 2014.

24. Jehle J, Schweizer PA, Katus HA and Thomas D: Novel roles for hERG K(+) channels in cell proliferation and apoptosis. Cell Death Dis 2: e193, 2011.

25. Liu J, Dayi HU, Liu W, Li C, Qin X, Li Y, Li Z, Li L, Dong W, Qi Y and Wang Q: Clinical characters and mutation analysis of potassium channel genes $\mathrm{KCNH} 2$ in 77 pedigrees of Chinese with long QT syndrome. Sci Technol Eng 11: 1529-1533, 2006 (In Chinese)

26. Li G, Mongillo M, Chin KT, Harding H, Ron D, Marks AR and Tabas I: Role of ERO1-alpha-mediated stimulation of inositol 1,4,5-triphosphate receptor activity in endoplasmic reticulum stress-induced apoptosis. J Cell Biol 186: 783-792, 2009.

27. Lam M, Dubyak G, Chen L, Nunez G, Miesfeld RL and Distelhorst CW: Evidence that BCL-2 represses apoptosis by regulating endoplasmic reticulum-associated $\mathrm{Ca}^{2+}$ fluxes. Proc Natl Acad Sci USA 91: 6569-6573, 1994.

28. Ma S, Zhao Y, Wei Y and Sun C: GW28-e0322 increasing calpain expression regulates hERG mutation L539fs/47 induced cardiomyocyte apoptosis. J Am Coll Cardiol 70: C6-C7, 2017.

This work is licensed under a Creative Commons Attribution-NonCommercial-NoDerivatives 4.0 International (CC BY-NC-ND 4.0) License. 